\title{
MiR-646 inhibited cell proliferation and EMT-induced metastasis by targeting FOXK1 in gastric cancer
}

P Zhang ${ }^{1}$, W M Tang ${ }^{1}$, H Zhang ${ }^{1,2}, Y$ Q Li ${ }^{1}, Y$ Peng ${ }^{1}$, J Wang ${ }^{1}, \mathrm{G} \mathrm{N} \mathrm{Liu}{ }^{1}, X$ T Huang ${ }^{1}$, J J Zhao ${ }^{3}, \mathrm{G} \mathrm{Li}^{4}, \mathrm{~A} \mathrm{M} \mathrm{Li}^{1}$,

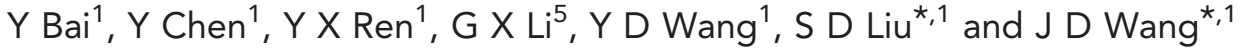

${ }^{1}$ Guangdong Provincial Key Laboratory of Gastroenterology, Department of Gastroenterology, Nanfang Hospital, Southern Medical University, Guangzhou 510515, China; ${ }^{2}$ Department of Gastroenterology, Hexian Memorial Affiliated Hospital of Southern Medical University, Guangzhou 511400, China; ${ }^{3}$ Department of Rheumatism, Nanfang Hospital, Southern Medical University, Guangzhou 510515, China; ${ }^{4}$ Department of Otolaryngology-Head and Neck Surgery, Nanfang Hospital, Southern Medical University, Guangzhou 510515, China and ${ }^{5}$ Department of General Surgery, Nanfang Hospital, Southern Medical University, Guangzhou 510515, China

Background: MiR-646 has been reported to be aberrantly expressed in human cancers. However, the underlying molecular mechanisms of action of miR-646 in gastric cancer (GC) have not yet been investigated.

Methods: In vitro function of miR-646 in GC was evaluated using EdU assay, plate colony formation assay, and matrigel invasion assay. Real-time PCR or western blotting was performed to detect miR-646 and FOXK1 expressions. In vivo tumour growth and metastasis were conducted in nude mice.

Results: MiR-646 expression was downregulated in GC tissues compared with adjacent normal tissues. Low miR-646 expression is associated with malignant progression. Transient transfection of GC cells with miR-646 inhibited their growth and migration. Moreover, miR-646 influenced the expression of epithelial-mesenchymal transition (EMT)-associated proteins. TGF- $\beta 1$ treatment significantly suppressed the expression of miR-646 and overexpression of this microRNA counteracted the influence of the TGF$\beta 1$-induced EMT phenotype. In terms of the underlying mechanism, miR-646 directly targeted FOXK1. In vivo, it inhibited the FOXK1-mediated proliferation and EMT-induced metastasis. Consistently, inverse correlations were also observed between the expression of miR-646 and FOXK1 in human GC tissue samples. Furthermore, miR-646 regulated Akt/mTOR signalling after FOXK1.

Conclusions: miR-646 inhibited GC cell proliferation and the EMT progression in GC cells by targeting FOXK1.

Gastric cancer (GC) is the fourth most common cancer in the world and the second leading cause of cancer death worldwide (Shah and Ajani, 2010). Similar to the development of other cancers, gastric carcinogenesis is multifactorial, involving environmental, genetic and epigenetic components (Kaneko et al, 2002; Ibarrola-Villava et al, 2015). Gastric cancer is surgically curable at an early stage, but advanced disease at the metastatic stage is associated with a high mortality rate (Stock and Otto, 2005; Yolanda et al, 2015). Elucidation of the mechanisms underlying GC tumourigenesis and metastasis will aid in the search for novel diagnostic biomarkers and the development of effective therapeutic interventions (Wagner and Moehler, 2009).

Increasing evidence indicates that microRNAs (miRNAs) function as oncogenes and tumour suppressors in cancer initiation,

*Correspondence: Professor S Liu; E-mail: liuside@163.com or Professor J Wang; E-mail: jidewang55@163.com

Received 10 December 2016; revised 26 March 2017; accepted 25 May 2017; published online 20 June 2017

(C) 2017 Cancer Research UK. All rights reserved 0007-0920/17 
progression and metastasis (Lu et al, 2005; Calin and Croce, 2006). MicroRNAs regulate gene expression by binding to the $3^{\prime}$ untranslated regions $\left(3^{\prime}\right.$-UTRs) of target mRNAs. Some studies have indicated that miR-646 is one of the important determinants in cancers (Sun et al, 2015; Pan et al, 2016). For example, abnormal expression of this miRNA in renal cell carcinoma has been shown to be associated with tumourigenesis and development ( $\mathrm{Li}$ et al, 2014). However, its role in GC proliferation and metastasis and the molecular mechanisms by which it regulates these processes are unknown.

Forkhead-box K1 (FOXK1), a forkhead family transcription factor, is involved in development and metabolism (Garry et al, 2000; Yang et al, 2000). Yang et al (2000) have shown that MNF/ Foxk1 recognises the DNA sequence motif WRTAAAAYA and regulates expression of the $p 21, c-m y c$, and $c d c 2$ genes (Wang et al, 2015). We have recently demonstrated that FOXK1 induces the epithelial-mesenchymal transition (EMT) in tumour cells, maintains the invasive potential of GC and appears to have a crucial role in the metastatic progression of human carcinomas (Peng et al, 2016). We have developed an algorithm to predict the targeting of FOXK1 by miR-646. However, whether this miRNA participates in the regulation of FOXK1 expression in GC remains unknown.

In this study, we showed that miR-646 expression was significantly downregulated in GC cell lines and tissues. Ectopic expression of miR-646 led to inhibition of GC cancer cell proliferation, migration, and invasion and reversal of the EMT. Moreover, miR-646 directly regulated the expression of FOXK1 by inducing EMT. Thus, we have revealed that miR-646 has regulatory effects on FOXK1 expression by activating Akt/mTOR signalling in GC; thus, this pathway could be a potential therapeutic target in cancer therapy.

\section{MATERIALS AND METHODS}

Cell culture and reagents. Human GC cell lines were purchased from the Type Culture Collection of the Chinese Academy of Sciences (Shanghai, China). The human normal gastric mucosal cell line GES-1 was acquired from Biowit Technologies Corporation (Shenzhen, China). The human gastric cell lines were cultured as described previously (Wang et al, 2007).

See Supplementary Information for more details.

Patients and specimens. Seventy-four GC tissues and 74 adjacent non-tumour tissues collected from GC patients between April 2014 and December 2015 were selected from the Department of Surgery of Nanfang Hospital, Southern Medical University, China. The Ethics Committee of the Southern Medical University approved our experimental protocols. All GC cases were confirmed by a senior pathologist and staged based on the 2011 Union for International Cancer Control TNM classification of malignant tumours. The pathological diagnoses of all enroled patients were confirmed by two different pathologists according to the WHO grading system (Torii et al, 1997).

ISH and IHC analyses. See Supplementary Information for more details.

Bioinformatics. The miRNA target prediction algorithms miRDB (http://mirdb.org/miRDB/), TargetScan (http://www.targetscan.org/), and microRNA (http://www.microrna.org/microrna/home.do) were used to predict miRNAs targeting FOXK1, as well as the miRNAbinding regions.

Oligonucleotide transfection. A miR-646 mimics $\left(40 \mathrm{nmoll}^{-1}\right.$ per well), miR-646 inhibitor $\left(100 \mathrm{nmoll}^{-1}\right.$ per well ) or their corresponding controls (m-NC for mimics and i-NC for inhibitor) were transfected using Lipofectamine 2000 transfection reagent (Invitrogen, Foster City, CA, USA) according to the manufacturers' protocols. Transfection efficiency was evaluated by a real-time PCR.

Western blot analysis. See Supplementary Information for more details.

RNA isolation and real-time qRT-PCR. See Supplementary Information for more details.

EdU incorporation assay. See Supplementary Information for more details.

Colony formation assay. See Supplementary Information for more details.

In vitro migration and invasion assays. See Supplementary Information for more details.

Luciferase activity assay. See Supplementary Information for more details.

Construction and transfection of lentivial vectors in vivo. A miR-646 lentiviral expression vector (Ubi-MCS-SV40-Cherry) containing the red fluorescent protein gene (GeneChem Co., Ltd, Shanghai, China) was transfected into the lentiviral packaging cell line 293T. Then, $1 \mathrm{ml}$ viral supernatant containing $4 \mathrm{AgAg}$ polybrene was added to GC cell lines for stable transduction. After 14 days, puromycin-resistant cell pools were established. A pGC-FU-GFP-LVFOXK1-CT lentiviral expression vector was constructed containing a functional FOXK1-CT fragment without the $3^{\prime}$-UTR region. To obtain mir-646/FOXK1-co-expressing cells, $3 \mu \mathrm{l}$ of a concentrated FOXK1-CT lentiviral expression vector solution was added to miR-646 overexpressing GC cell lines. Five to approximately $8 \mu \mathrm{g} \mathrm{ml}^{-1}$ polybrene were then mixed with the cells. After $72 \mathrm{~h}$, western blotting was performed to detect FOXK1 expression.

Animal models. BGC-823 cells $\left(5 \times 10^{6}\right)$ transfected with an NC, miR-646, or miR-646/FOXK1 lentivirus were suspended in $100 \mu \mathrm{l}$ serum-free RPMI and implanted subcutaneously into the flanks of nude mice (three 4-6-week-old female BALB/c nu/nu mice in each group; Laboratory Animal Unit, Southern Medical University, China). Then, the fluorescence emitted by cells was collected and imaged using a whole-body RFP (NC-RFP and miR-646-RFP) or GFP (miR-646/FOXK1-GFP) imaging system (Lighttools, Encinitas, CA, USA). The sizes of the resulting tumours were measured weekly. Tumour volumes were calculated as follows: total tumour volume $\left(\mathrm{mm}^{3}\right)=L \times W^{2} / 2$, where $L$ is the length and $W$ is the width. The mice were monitored for weight loss and mortality for 25 days. Mice that lost more than $20 \%$ of their body weight were humanely killed. Their tumours were dissected and weighed.

For tail vein metastasis assay, a total of $5 \times 10^{6}$ cells were injected into the tail veins of nude mice. After 40 days, the mice were killed and lung tissues were dissected and subjected to histological examination. Metastatic tumours were detected by $\mathrm{H} \& \mathrm{E}$ staining and were quantified by the counting of metastatic lesions in each section.

Statistical analysis. All statistical analyses were performed using the SPSS 20.0 software (SPSS Inc., Chicago, IL, USA) and data were expressed as the mean \pm s.d. $P<0.05$ was considered statistically significant. The relative quantification of gene expression detected by real-time PCR was $\log 2$ transformed and analysed by Student's $t$-test. Linear or rank correlation analysis was performed to determine the correlation between the gene expression levels. Pearson's $\chi^{2}$-test was used to analyse the associations of miR-646 expression with clinicopathologic features. For cell line experiments and animal assays, data were subjected to a two-tailed Student $t$-test or one-way ANOVA ( $T$-test for two-group comparisons, otherwise one-way ANOVA). 


\section{RESULTS}

MiR-646 expression is markedly down-regulated in GC cell lines and tissues. First, we assessed the expression of miR-646 in five GC cell lines (MKN28, BGC-823, MGC-803, AGS, and HGC-27) by quantitative PCR. The results revealed that the expression of miR-646 was substantially decreased in all of the GC cell lines compared with a transformed human gastric epithelial cell line (GES-1) (Figure 1A).

Moreover, we examined the expression of miR-646 in 74 pairs of human GC tissues and matched non-tumour tissues. The results revealed that expression of this miRNA was down-regulated by up to 534 -fold in 55 of the 74 GC samples compared with the control samples (Figure 1B). Its expression was significantly lower in the GC tissues compared with the adjacent normal tissues in patients by RT-PCR (Figure 1C). Furthermore, in situ hybridisation (ISH) revealed that it was localised in both the nuclei and cytoplasm of GC cells, as shown in Figure 1D.

These findings demonstrate that miR-646 may act as a tumour suppressor in GC.
MiR-646 expression attenuates the malignant biological behaviour of GC. To determine the clinical relevance of miR-646 expression, we assessed the clinicopathological features in GC. No significant association was observed between miR-646 expression and age $(P=1.000)$, gender $(P=1.000)$, or differentiation $(P=0.530)$. However, its expression was significantly correlated with tumour size $(P=0.034)$, tumour invasion $(P=0.01)$, lymph node metastasis $(P=0.001)$, and TNM stage (AJCC) $(P=0.01)$ (Supplementary Table 1$)$. These results show that low miR-646 expression is associated with malignant progression in GC patients.

To assess the role of miR-646 in the development of GC, we transfected GC cells with miR-646 mimics or a miR-646 inhibitor; miR-646 expression was confirmed by qRT-PCR (Supplementary Figure $1 \mathrm{~A}$ and $\mathrm{B})$.

The results of CCK- 8 assays revealed that miR-646 could significantly decrease cell proliferation in BGC-823, AGS, MKN28, and MGC-803 $(P<0.001$, Supplementary Figure $1 C)$. EdU assay showed that the proliferation rate of GC cells transfected with miR646 mimics was significantly decreased compared with that of cells transfected with negative control mimics (m-NC). In contrast, the
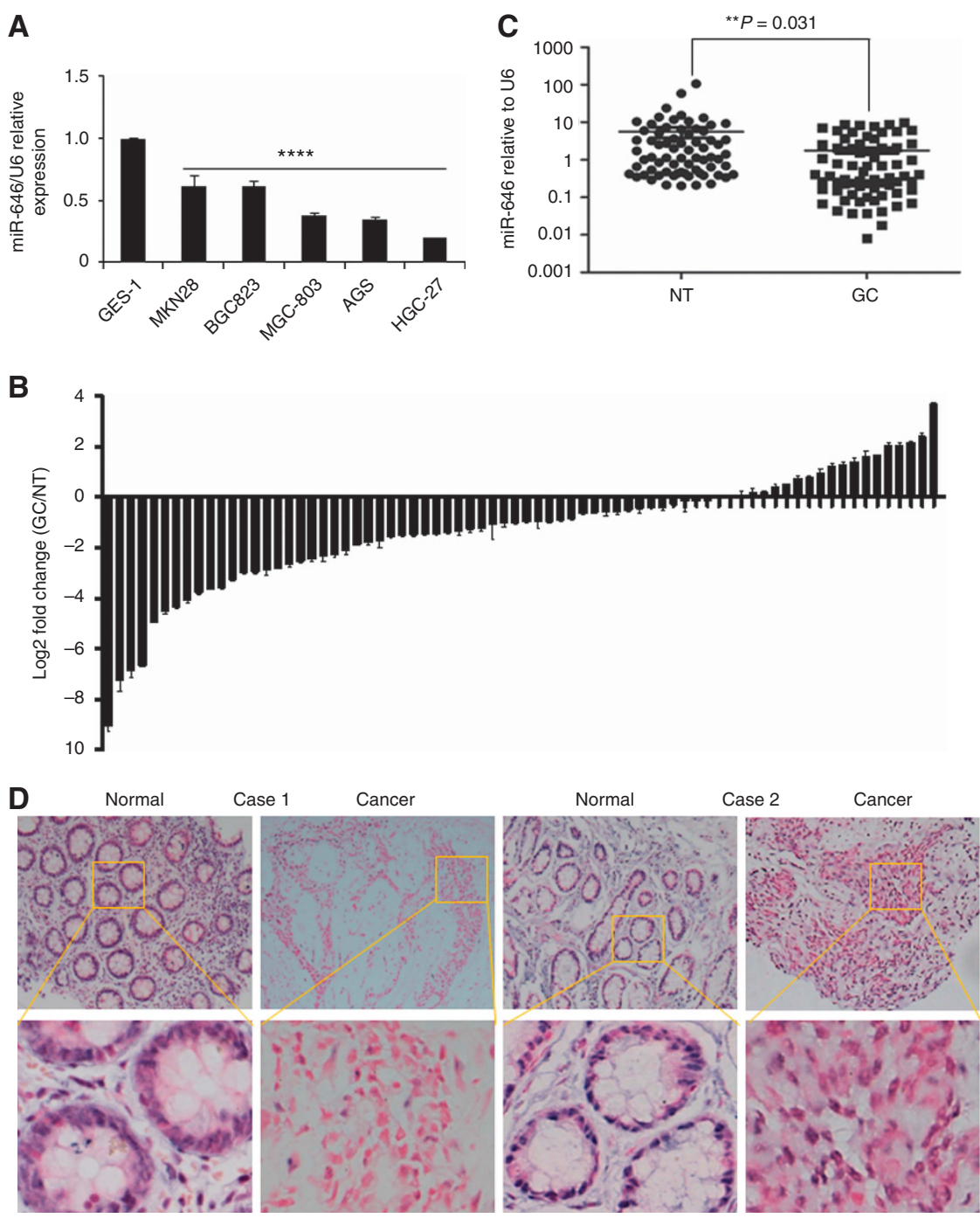

Figure 1. miR-646 expression is downregulated in GC cell lines and tissues. (A) Decreased miR-646 expression was detected in all five GC cell lines compared with the normal human gastric epithelial cell line GES-1. ${ }^{\star \star \star \star} P<0.001$. (B) Real-time PCR analysis of miR-646 expression in 74 pairs of human GC tissues and their adjacent normal mucosal tissues. The error bars represent the mean \pm s.d. from three independent experiments. (C) As analysed by qRT-PCR, miR-646 expression level in GC tissues was significantly lower than that observed in the corresponding noncancerous gastric mucosa tissue. ${ }^{* \star} P<0.05$ (D) Representative ISH images of miR-646 expression. Scale bars, $50 \mu \mathrm{m}$. 
proliferation rate of GC cells transfected with the miR-646 inhibitor was significantly increased compared with that of cells transfected with a negative control inhibitor (i-NC, Figure 2A, $P<0.001)$.

Next, colony-formation assay showed that cells transfected with miR-646-mimic yielded significantly fewer colonies compared with those transfected with m-NC. Conversely, transfection of cells with the miR-646 inhibitor had the opposite effect, resulting in an increased number of colonies compared with that of cells transfected with i-NC, as predicted (Figure $2 \mathrm{~B}$ ). Taken together, these results demonstrate that the increased expression of miR-646 in GC cells results in the inhibition of cell proliferation and transformation.

Furthermore, to evaluate the roles of miR-646 in cell migration and invasion, wound healing and transwell invasion assays were performed using GC cells. Wound-healing assay showed that the miR-646 mimics markedly inhibited GC cell migration. Consistent with this result, the miR-646 inhibitor significantly increased GC cell migration (Figure 2C). Transwell invasion assays indicated that the invasiveness of GC cells transfected with miR-646 mimics was significantly decreased, whereas it was increased in those transfected with the miR-646 inhibitor (Figure 2D).

These results indicate that miR-646 regulates the malignant biological behaviour of GC cells.
MiR-646 inhibits the EMT in GC cells. The EMT is recognised as an important process that occurs during GC cell invasion ( $\mathrm{Wu}$ et al, 2016a,b,c,d; Yang et al, 2008). To determine whether miR-646 has a critical role in the GC EMT, we first examined cell morphology. We observed that miR-646 expression caused the transformation of GC cells into spheroids with few protrusions (negative control inhibitor, i-NC), whereas inhibition of its expression caused the GC cells to undergo spindle-like morphological changes (Figure 3A). These findings suggested that some molecular changes might have occurred during alteration in miR646 expression.

We next visualised the actin cytoskeleton by phalloidin staining. In contrast with $\mathrm{i}-\mathrm{NC}$, the miR-646 inhibitor caused a change in the F-actin distribution in these cells from a cortical to a stressfibre pattern, a hallmark of the mesenchymal phenotype (Supplementary Figure 2A).

Finally, we assessed the expression of EMT markers by immunofluorescence analysis after miR-646 inhibition. The results revealed that the expression of E-cadherin was downregulated, whereas that of vimentin was upregulated (Supplementary Figure 2B). In addition, western blot analysis showed that the expression of EMT-associated markers was altered by transfection of miR-646 mimics or inhibitors. MiR-646 induction increased the level of the epithelial marker E-cadherin and suppressed the levels
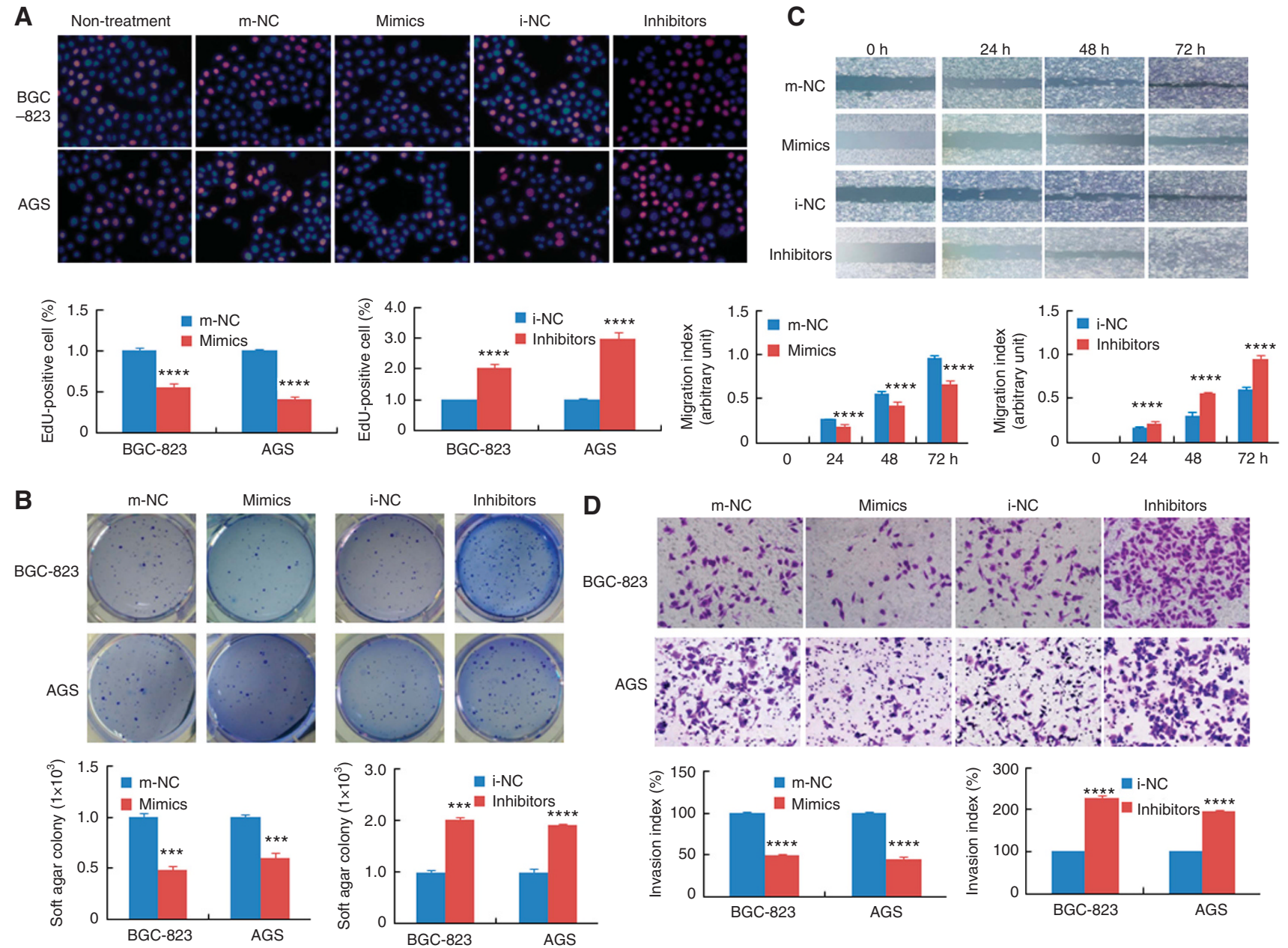

Figure 2. Functional analysis of miR-646 in vitro. (A) DNA synthesis in GC cells was measured by EdU incorporation assay at $48 \mathrm{~h}$ after the indicated transfection. Red fluorescence represents the EdU-positive cells; blue fluorescence from the Hoechst stain represents the total cells. $\star \star \star \star P<0.001$, mimics vs $\mathrm{m}-\mathrm{NC}$; ${ }^{\star * \star *} P<0.001$, inhibitor vs i-NC. (B) Effects of miR-646 mimics or inhibitor on the proliferation of GC cell lines, as determined by anchorage-independent growth ability assay. ${ }^{\star \star \star} P<0.01$. (C) Cell migration activity was measured by wound healing assay at $48 \mathrm{~h}$ after transfection with miR-646. ${ }^{* \star \star *} P<0.001$ and ${ }^{* \star \star} P<0.01$. (D) To evaluate cell invasiveness, transwell assays were performed on miR-646. Cells were counted under a microscope in five randomly selected fields. ${ }^{\star \star \star \star} P<0.001$. Scale bars, $100 \mu \mathrm{m}$ in $\mathbf{A}$. 

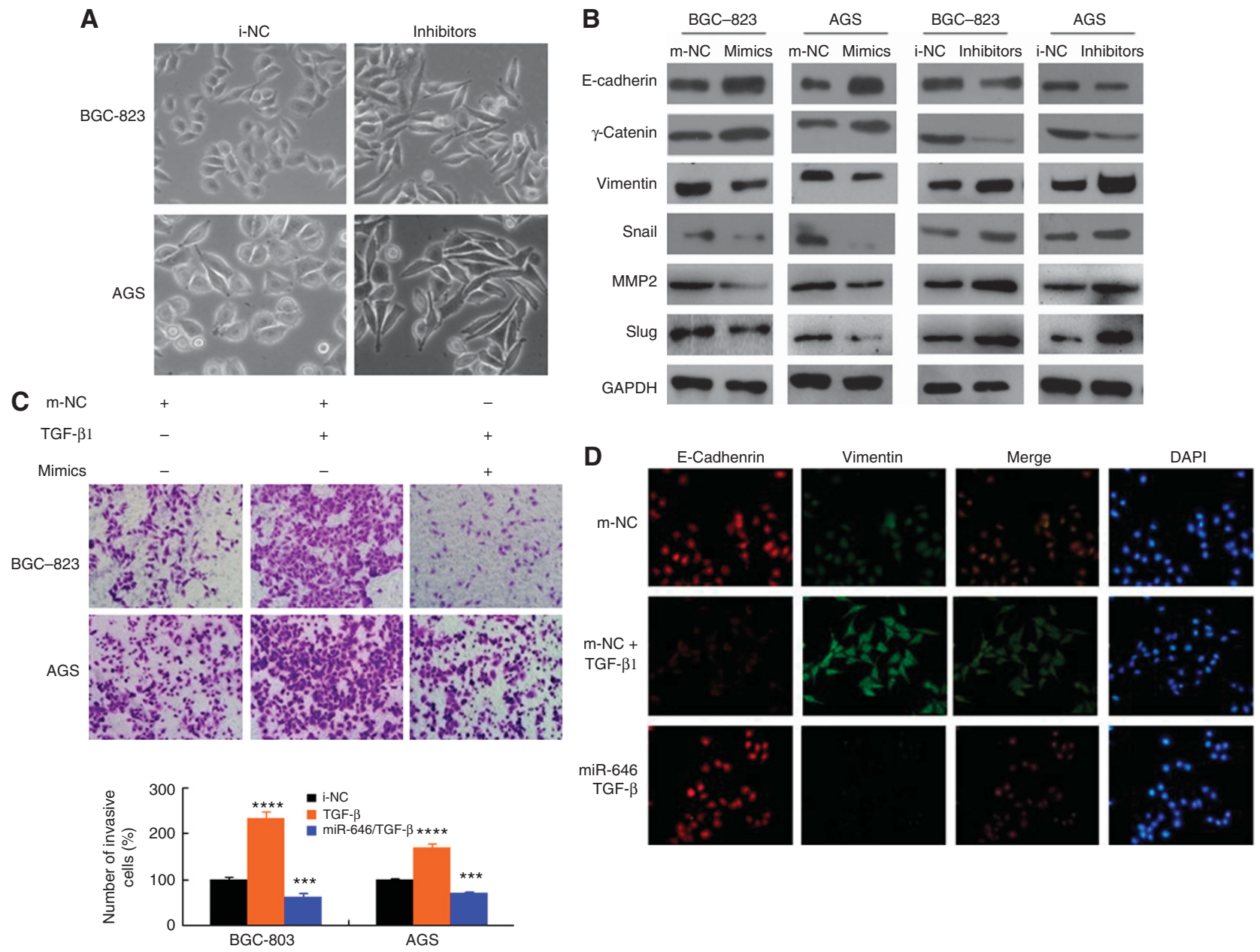

Figure 3. miR-646 inhibits the EMT in TGF- $\beta$-treated GC cells. (A) Morphology of the miR-646 expression levels with i-NC and inhibitor cells, as visualised by phase-contrast microscopy. (B) The expression of EMT biomarkers, including E-cadherin, s-catenin, vimentin, Snail, MMP2, and Slug, was detected by western blotting at $48 \mathrm{~h}$ after transfection. (C) Representative figures and data from transwell assays performed using GC cells treated with miR-646 mimics and/or TGF- $\beta\left(5 \mathrm{ng} \mathrm{ml}^{-1}\right)$ for $24 \mathrm{~h}$. ${ }^{\star \star \star} P<0.01$ and ${ }^{\star \star \star \star \star} P<0.001$. (D) Immunofluorescence staining for the expression of E-cadherin (red) and vimentin (green) in BCG-823 cells treated with miR-646 mimics and TGF- $\beta\left(5 \mathrm{ng} \mathrm{ml}^{-1}\right)$, TGF- $\beta$ only or m-NC. DAPI was used to show the locations of nuclei (blue). Representative immunofluorescence images were captured. The scale bars represent $20 \mu \mathrm{m}$ in $\mathbf{A}$ and $\mathbf{D}$.

of the mesenchymal markers vimentin, snail, MMP2, and Slug. In contrast, miR-646 inhibition had the complete opposite effect (Figure 3B). These data demonstrate that miR-646 deficiency in GC cells might be an important contributor to EMT progression, thereby facilitating the migration and invasion of GC cells in vitro.

MiR-646 attenuates the EMT in TGF- $\beta$ 1-treated GC cells. The EMT is reportedly induced by various signals in the tumour microenvironment, including TGF- $\beta 1$ (Wu et al, 2016a,b,c,d; Hou et al, 2016). We first examined the miR-646 levels in TGF- $\beta 1$ treated BGC-823 and AGS cells. Notably, TGF- $\beta 1$ treatment resulted in a significant dose- and time-dependent decrease in miR-646 expression (Supplementary Figure 2C). Moreover, we ectopically expressed miR-646 in GC cells and then treated the cells with TGF- $\beta 1$. Ectopic expression of this miRNA partly inhibited the EMT-like phenotypic changes of the cells (Supplementary Figure 2D). In addition to promoting EMT-related morphological changes, miR-646 mimics significantly suppressed the effects of TGF- $\beta 1$ on invasiveness of the tumour cells, as determined by transwell assay (Figure 3C).

Furthermore, immunofluorescence staining showed that miR646 overexpression reversed the TGF- $\beta 1$-mediated upregulation of vimentin and downregulation of E-cadherin (Figure 3D).
Collectively, these data suggest that miR-646 attenuates the TGF- $\beta 1$-induced EMT of GC cells.

FOXK1 is a direct downstream target of miR-646. To explore the molecular mechanisms by which miR-646 regulates GC cell growth and metastasis, the miRDB, TargetScan, and miRNA databases were used to predict targets of miR-646 and FOXK1 was identified as a potential target. Next, we performed luciferase reporter assay to determine whether FOXK1 was a direct target of miR-646 in GC cells. The target region sequence of the FOXK1 3'UTR (WT $3^{\prime}$-UTR) or a mutant sequence containing two putative miR-646 sites (Mut 1 or Mut $23^{\prime}$-UTR) was cloned into a luciferase reporter vector (Figure $4 \mathrm{~A}$ ). These constructed reporter vectors were then cotransfected with miR-646 mimics or m-NC into the GC cell lines. Our findings indicated that miR-646 decreased the luciferase activity of the FOXK1 WT $3^{\prime}$-UTR construct. Mutation of either of the two sites abolished the inhibitory effect of miR-646 on luciferase activity (Figure 4B).

To further confirm that FOXK1 is a target gene of miR-646, western blot analysis was performed to determine whether FOXK1 protein expression was influenced. The results revealed that expression of this protein was down-regulated in the cells transfected with miR-646 mimics compared with the NC cells but that it was inversely upregulated in the cells transfected with 
A Wild-type 3' UTR 5' UGCUCACAUGUG CUG CUA 3 (site 1:5062-5068 nt) ||| || | has-mIR-646: 3'CGGAGUCUCCGU CGACGAA 5' Mutant $13^{\prime}$ UTR 5, GCUCACAUGUGAUUCGA 3',

B

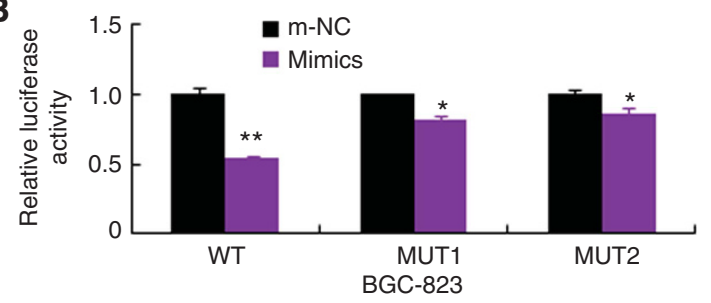

C

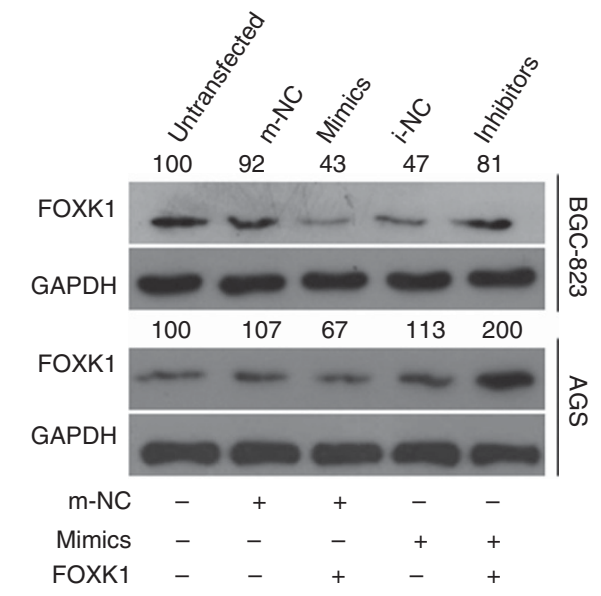

E

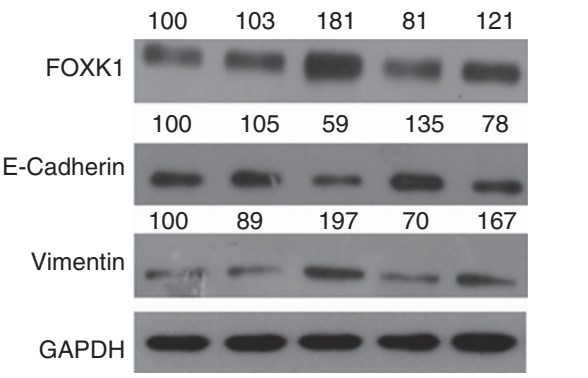

Wild-type 3' UTR 5' UUGAUAC GAAAAGCUG CUA3' (site 2 : 5331-5338 nt) has-mIR-646: 5 ' CGGAGUCUCCGU CGACGAA3' || $1111 \mid$ II | |

Mutant 2 3' UTR 5' UUGAUACGAAAAGAUUCGA 3'
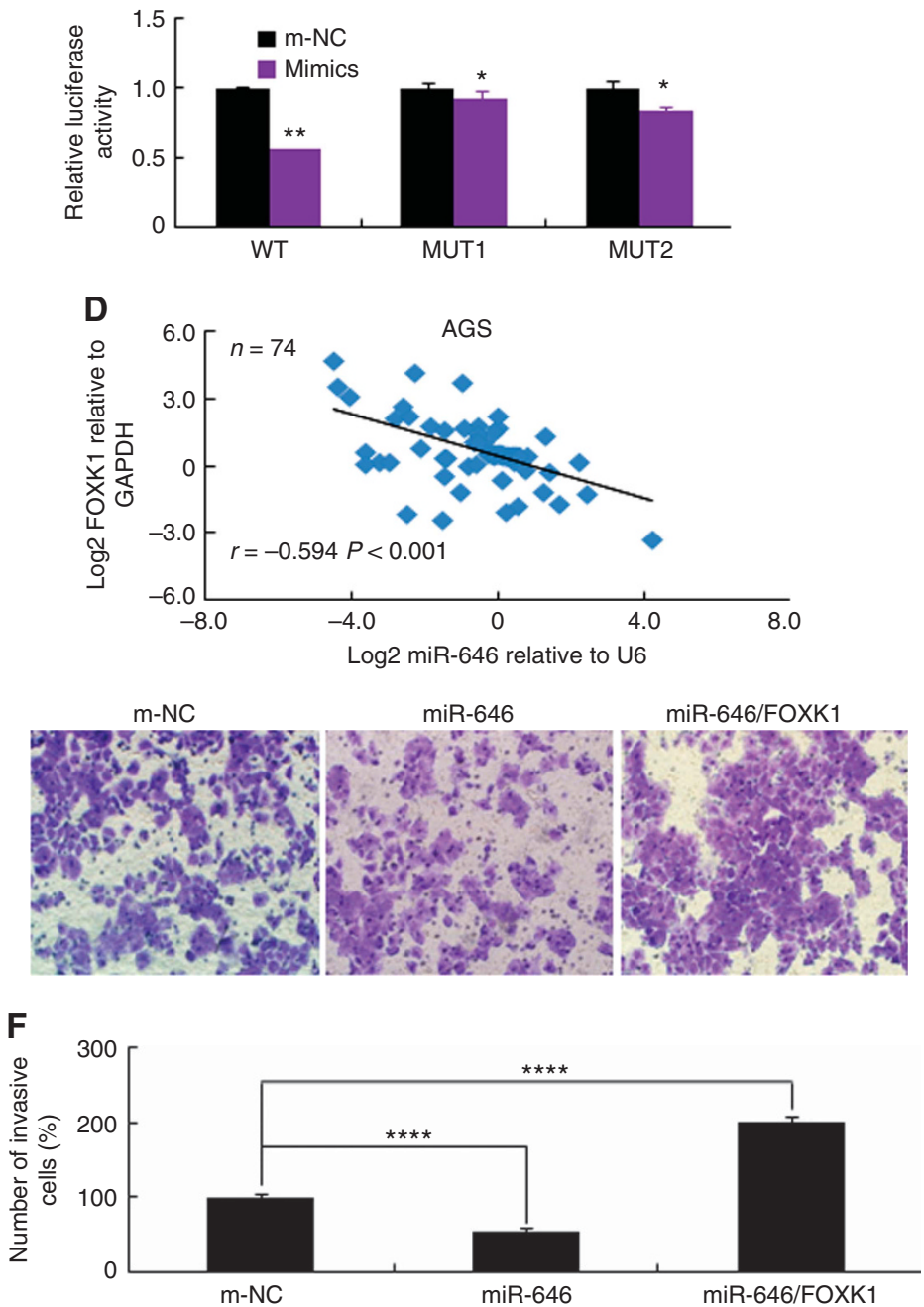

Figure 4. FOXK1 is a direct target of miR-646. (A) The putative miR-646-binding sites in the FOXK1 3'-UTR; nt, nucleotides. (B) Luciferase activity in the indicated GC cells following transfection of conserved miR-646 binding site-mutated 3'-UTR-driven reporter constructs; wt, wild type; mut, mutant; site 1, the miR-646 motif spanning nt 5062-5068 nt; site 2, the motif spanning nt 5331-5338 nt in the FOXK1 $3^{\prime}-U T R$; $n=3$. ${ }^{* \star} P<0.05$ and ${ }^{*} P>0.05$. (C) Western blot analysis of FOXK1 protein expression in GC cells transfected with miR-646 mimics or inhibitor. Untransfected cells, as well as cells transfected with a control mimic or inhibitor plasmid, were used as controls. (D) In human GC tissues, FOXK1 was negatively correlated with miR-646 at the mRNA level $(n=74)$. (E) The FOXK1, E-cadherin, and vimentin protein levels were detected by western blotting in cells transfected with a FOXK1 expression plasmid, miR-646 mimics, or m-NC. The relative protein expression levels were quantified by comparing the gray level of each band using Quantity One Software (Life Science Research, Hercules, CA, USA). (F) Migratory activity of cells transfected with a FOXK1 expression plasmid and/or miR-646 mimics was evaluated by transwell invasion assay. ${ }^{\star \star \star \star} P<0.001$. All of these experiments were repeated three times with identical findings.

the miR-646 inhibitor (Figure 4C). In human GC tissues, we found that the mRNA level of FOXK1 was negatively correlated to miR646 (Figure 4D), which suggested that a modulating effect existed between these two parameters. These data suggest that miR-646 might inhibit FOXK1 protein expression through the $3^{\prime}$-UTR at the posttranscriptional level.

To determine whether miR-646 affects the EMT by regulating FOXK1 expression, FOXK1 was reintroduced into miR-646 mimic-transfected BGC-823 cells. Although miR-646 increased E-cadherin expression and decreased vimentin expression, FOXK1 induction significantly ameliorated these changes by suppressing E-cadherin expression and promoting vimentin expression (Figure 4E). These molecular changes also influenced GC cell function, as the suppression of invasiveness by miR-646 was reversed by FOXK1 induction (Figure 4F). In summary, these data indicate that miR-646 deficiency results in the upregulation of FOXK1 expressions, which in turn mediates the EMT in GC.

MiR-646 regulated the FOXK1-mediated proliferation in GC cells in vivo. To assess the effect of miR-646 on tumour growth in vivo, BGC-823/miR-646 cells, BGC-823/miR-646/FOXK1 cells, or m-NC cells were implanted subcutaneously into nude mice, and the growth of resultant primary tumours then was monitored. The mice injected with BGC-823/miR-646 cells developed smaller tumours than those injected with miR-646/FOXK cells. The tumours of the mice injected with miR-646/FOXK cells 


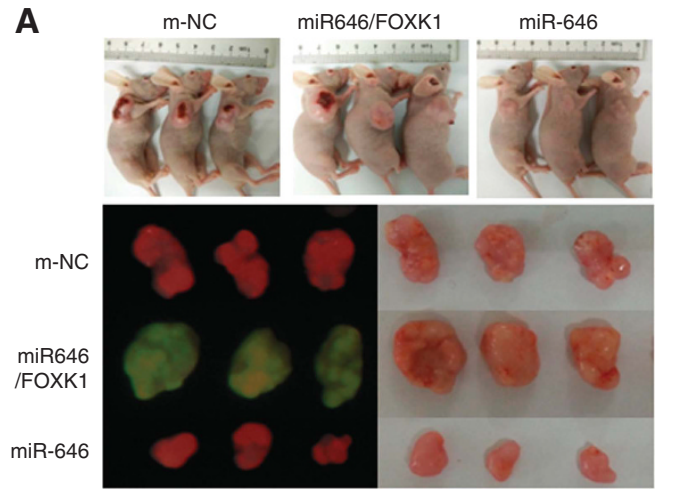

B
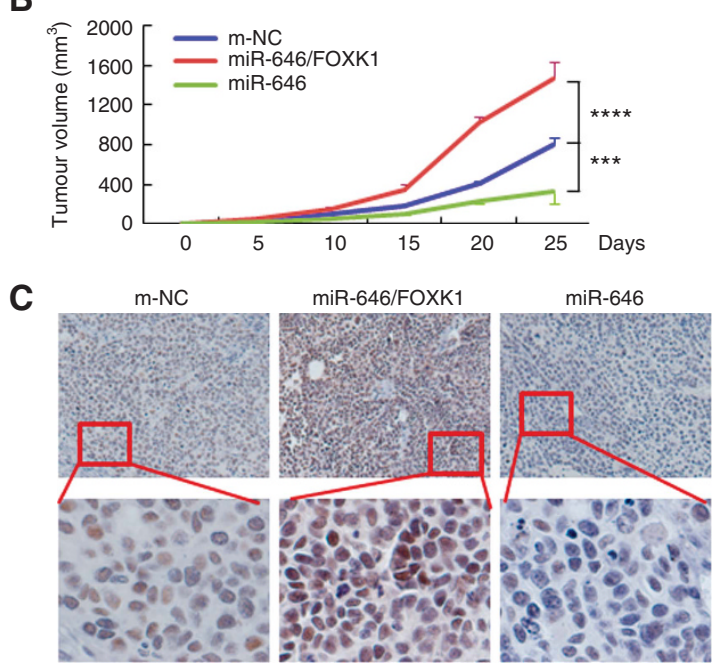

$\mathbf{E}$
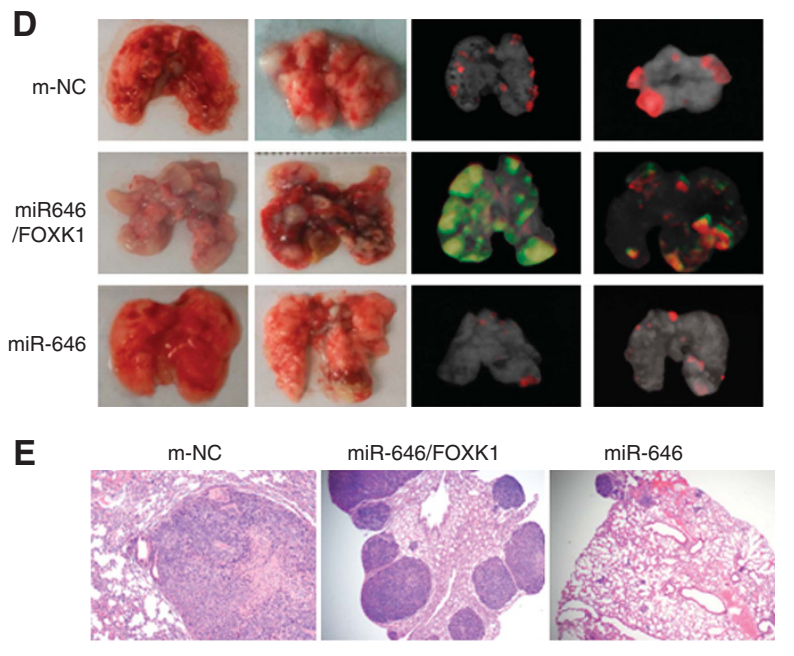

$\mathbf{F}$

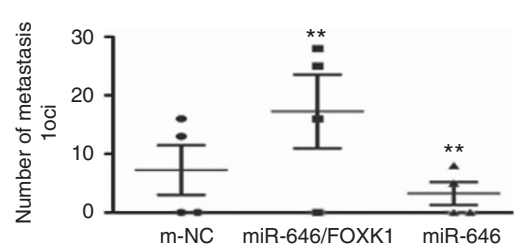

G

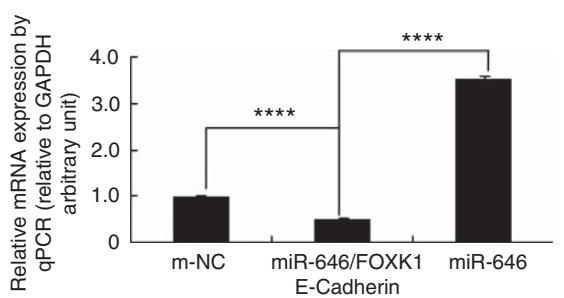

Figure 5. miR-646 regulated GC tumour growth and metastasis by targeting FOXK1 in vivo. (A) Fluorescence images of subcutaneous tumours from mice injected with BGC-823/m-NC, BGC-823/miR-646/FOXK1, or BGC-823/miR-646 cells. (B) Tumour size was measured at 5 days after tumour cell inoculation in each group. ${ }^{\star \star \star} P<0.01$, $\mathrm{m}-\mathrm{NC}$ vs miR-646/FOXK1; and ${ }^{\star \star \star \star} \mathrm{P}<0.001$, miR-646 vs miR-646/FOXK1. (C) Immunohistochemical (IHC) staining of FOXK1 expression in subcutaneous tumours from mice injected with BGC-823/m-NC, BGC-823/miR-646/FOXK1, or BGC-823/miR-646 cells. (D) Mice were orthotopically transplanted with BGC-823 cells ( $n=3$ in each group). (E) Metastatic cancer tissues were stained with H\&E. (F) The number of metastatic loci in the lungs was counted. ${ }^{* *} P<0.05, \mathrm{~m}-\mathrm{NC}$ vs miR-646/FOXK1 and miR-646/FOXK1 vs miR-646. (G) The expression of E-cadherin in tumours derived from BGC-823 cells was determined by quantitative PCR. ${ }^{\star \star \star \star} P<0.001, \mathrm{~m}-\mathrm{NC}$ vs miR-646/FOXK1; miR-646 vs miR-646/FOXK1. Scale bars, $200 \mu \mathrm{m}$ in C and $400 \mu \mathrm{m}$ in E.

significantly differed from those of the mice injected with m-NC or miR-646 cells (Figure 5A and B). Immunohistochemical (IHC) staining verified the positive expression of FOXK1 in the miR-646/ FOXK-xenografted tumours compared with its negative expression in the miR-646-xenografted tumours (Figure 5C). These data confirmed that MiR-646 regulated the FOXK1-mediated growth in GC.

MiR-646 inhibited the FOXK1-mediated metastasis via decreased EMT in vivo. To determine the effect of miR-646 on GC metastasis in vivo, miR-646, miR-646/FOXK1, or m-NC cells were injected into nude mice via the tail vein, which resulted in lung metastasis within forty days. The organs of the mice were subsequently scanned for metastasis using a whole-body visualisation system (Figure 5D). MiR-646/FOXK1 cells, but not m-NC or miR-646 cells, formed a number of large metastatic nodules in the lungs (Figure 5D). Gastric cancer metastasis to the lungs was confirmed by histological analysis (Figure 5E). Compared with miR-646/FOXK1 cells, miR-646 and m-NC cells exhibited significant decreases in visible lung tumours, and these decreases were correlated with reduced numbers of metastatic loci (Figure 5F).

We next showed that whether miR-646 affected the EMT process by regulating FOXK1 expression. Although miR-646 increased E-cadherin expression, miR-646/FOXK1 induction significantly ameliorated these activities by suppressing E-cadherin expression (Figure 5G). These results showed that miR-646 deficiency upregulated FOXK1, which in turn mediated the EMT in GC.

Based on these results, it is reasonable to conclude that miR-646 inhibited the FOXK1-mediated metastasis through decreased EMT in GC cells in vivo.

MiR-646 represses EMT and Akt/mTOR signalling by directly targeting FOXK1. To explore whether FOXK1 cooperates with miR-646 in GC tissues EMT modulation, ISH was performed to detect miR-646 expression, and IHC analysis was conducted to analyse the FOXK1 and E-cadherin protein levels. The results confirmed that miR-646 caused significant upregulation of expression of the epithelial marker E-cadherin expression, while FOXK1 downregulated of expression in normal gastric tissues. On the contrary, FOXK1 protein was high-expressed in the carcinoma cells of all GC samples. MiR-646 and E-cadherin expression was inversely associated with FOXK1 as exemplified in Figure 6A.

Previous studies have shown that AKT/mTOR signalling induces the EMT (Chang et al, 2013; Wang et al, 2016). In the present study, we found that knockdown of FOXK1 by siRNA in 

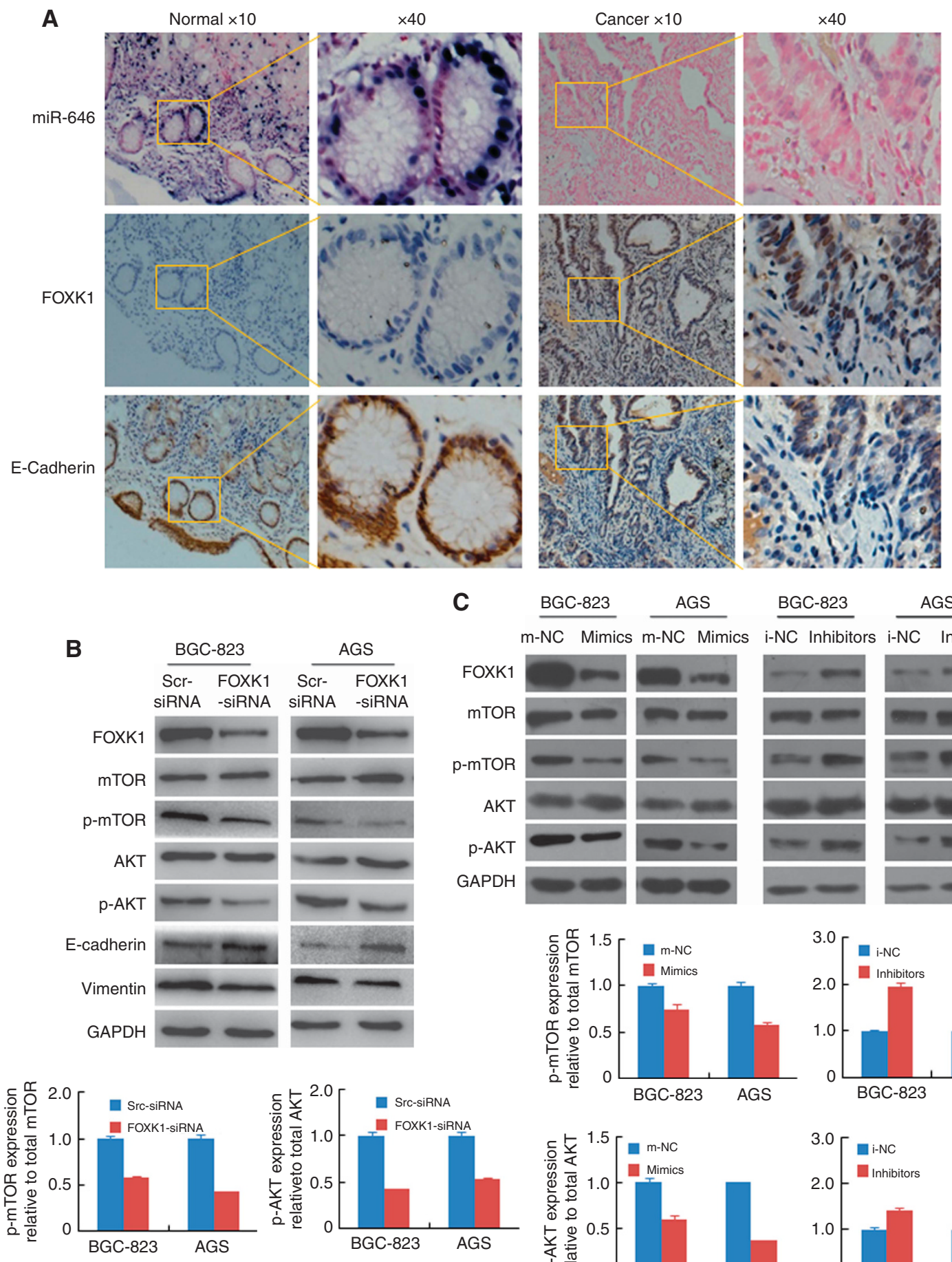

B

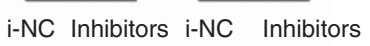

m-NC Mimics m-NC Mimics i-NC Inhibitors i-NC Inhibitors

mTOR
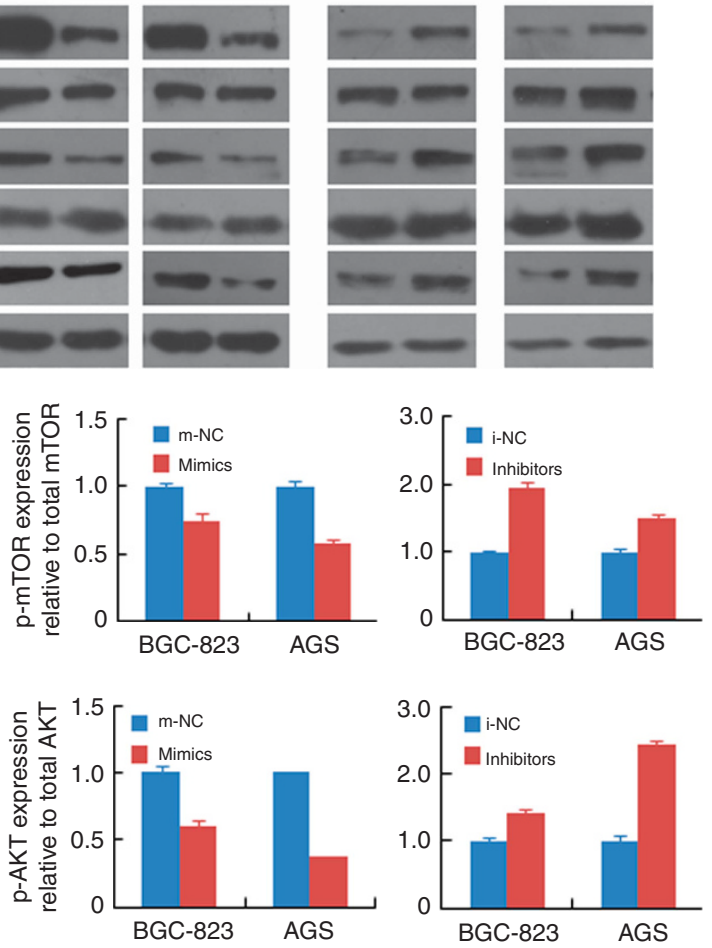

Figure 6. miR-646 suppresses the EMT via Akt/mTOR signalling. (A) ISH analysis of miR-646 and IHC analysis of FOXK1 and E-cadherin were performed. These figures were the representatives of gastric tissues from 10 cancerous and 10 non-cancerous patients. The scale bars represent $200 \mu \mathrm{m}$ in A. (B) Knockdown of FOXK1 by siRNA in gastric cancer cell decreased the protein expression of E-cadherin, vimentin and phosphorylation of Akt and mTOR. (C) FOXK1, mTOR, p-mTOR, Akt, and p-Akt protein expression in BGC-823 and AGS cells transfected with the miR-646 mimic, inhibitor, or corresponding control (NC) plasmid.

gastric cancer cell decreased the protein expression of E-cadherin, vimentin and phosphorylation of Akt and mTOR (Figure 6B). Moreover, the levels of both phosphorylated Akt and phosphorylated mTOR were decreased in the miR-646 mimic-transfected GC cells but that they were elevated in the miR-646 inhibitortransfected cells. In addition, FOXK1 expression counteracted the downregulation of p-Akt and p-mTOR induced by miR-646 (Figure 6C).
These results suggest that miR-646 suppresses FOXK1 expression and helps to stabilise the epithelial phenotype in human GC.

\section{DISCUSSION}

In this study, the role of miR-646 in GC cell growth and migration was characterised. Low miR-646 levels were detected in the 
majority of GC tissues examined compared with those in nontumour tissues. Moreover, miR-646 expression was significantly correlated with adverse clinicopathological factors. Ectopic expression of miR-646 suppressed GC cell proliferation, migration, and invasion both in vitro and in vivo. We further explored the underlying mechanisms and found that this miRNA inhibited GC progression by directly targeting the FOXK1 and Akt/mTOR signalling pathways. These results demonstrate that miR-646 has a vital role in regulating GC development and progression.

Although previous studies have detected miR-646 in multiple tumours, including lung cancer and kidney tumours (Li et al, 2014; Pan et al, 2016), little is known about its role in carcinogenesis and progression in GC. In the current study, we evaluated miR-646 expression in human GC tumour tissues and cell lines. We also explored the biological role and regulatory mechanism of miR-646 in GC for the first time, confirming that its expression was downregulated in GC tumour tissues compared with non-tumour tissues. Moreover, its expression was significantly correlated with adverse clinicopathological factors, such as tumour size, tumour invasion, lymph node metastasis, and TNM stage. These strong correlations suggest that miR-646 ectopic expression may inhibit tumour invasion and metastasis. The results of this study confirm the tumour-suppressive role of miR-646 in GC cells and provide evidence of the potential utility of this miRNA for miRNA-based cancer therapy. The EMT, one of the central mechanisms that promote tumour invasion and metastasis, is a process by which epithelial cells lose their polarity and are converted to a mesenchymal phenotype (Kim et al, 2011; Hur et al, 2013; Wang et al, 2016). The EMT is associated with loss of the cell-cell adhesion molecule E-cadherin and disruption of cell-cell junctions, as well as the acquisition of migratory properties. Previous studies have suggested that the miR-15/107 group of miRNA genes regulate the EMT and migration invasion of tumours (Martello et al, 2010; Wang et al, 2014; Liu et al, 2015; Dong et al, 2016; Dwivedi et al, 2016; Wu et al, 2016d). For example, miR-16 suppresses EMT-related gene expression in human glioma (Wang et al, 2014). In addition, miR-195 inhibits the EMT by targeting FGF2 in prostate cancer cells (Liu et al, 2015). Accordingly, we hypothesised that miR-646 is involved in EMT-mediated GC metastasis. We found that expression of this miRNA was closely associated with EMT marker levels. Through loss-of-function experiments, we demonstrated that miR-646 induces the EMT and metastatic phenotypes. Our results suggest that this miRNA likely participates in EMT-associated GC progression. Therefore, this study provides useful insights into the mechanisms underlying GC metastasis. The TGF- $\beta$ signalling pathway has pivotal roles in diverse developmental processes and the pathogenesis of many diseases, including cancer (Li et al, 2015; Peng et al, 2016; Wu et al, $2016 \mathrm{a}, \mathrm{b}, \mathrm{c}, \mathrm{d})$. Several studies have shown that miRNAs regulate downstream molecules of TGF- $\beta$ to modulate the EMT. For example, miR-125b has been reported to inhibit the EMT by targeting SMAD2 in HCC (Zhou et al, 2015). In addition, Hou et al (2016) have demonstrated that a negative feedback loop involving miR-340 and ZEB1 regulates TGF- $\beta$-mediated breast cancer progression. Further, recent studies have shown that miR-132/ 212 upregulation inhibits the TGF- $\beta$-mediated EMT of prostate cancer cells by targeting SOX4 (Fu et al, 2016). Moreover, Huang et al (2015) have reported that miR-451 functions as an EMT inhibitor by targeting the $\mathrm{c}-\mathrm{Myc} / \mathrm{Erk} 1 / 2$ axis. In this study, we observed that miR-646 expression decreased in a time- and dosedependent manner after treatment with TGF- $\beta$. TGF- $\beta$-mediated enhancement of cell migration and invasion was attenuated following incubation of cells with miR-646. Moreover, miR-646 reversed the EMT phenotype of GC cells. Thus, this miRNA appears to be a significant downstream inhibitor of TGF- $\beta$ signalling, which attenuates the proinvasive effects of TGF- $\beta$ in GC cells.
MiR-646 has many predicted targets, including oncogenes, such as FGF2, EGFR and NOB1 (Li et al, 2014). Forkhead-box K1, a member of the FOX family of transcription factors, has been implicated in certain tumour types, including colorectal, gastric and ovarian tumours (Ying et al, 2014; Peng et al, 2016; Wu et al, 2016a, b, c, d). Forkhead-box K1 has been shown to contribute to loss of the epithelial marker E-cadherin and upregulation of the expression of mesenchymal markers, including vimentin, and to promote tumour cell invasion (Wu et al, 2016a, b, c, d). In the present study, we have demonstrated that miR-646 suppresses FOXK1 expression; we believe that FOXK1 contribute to the inhibitory effect of miR-646 on the EMT in GC. In addition, miR646 expression suppressed the phosphorylation of AKT and mTOR. A previous study has shown that miR-193a-3p and 5p induce the EMT and metastasis by activating the AKT/mTOR signalling pathway in tumour cells ( $\mathrm{Yu}$ et al, 2015). These results suggest that dysregulation of the FOXK1AKT/mTOR signalling pathway through miR-646 is an important mechanism underlying tumour metastasis.

In summary, we have revealed that miR-646 expression is downregulated in GC tissues and that this downregulation is correlated with disease progression. MiR-646 suppresses the proliferation, migration and invasion of GC cells and inhibits the EMT. In addition, this miR-646 inhibits FOXK1 expression by directly binding to their $3^{\prime}$-UTRs. The FOXK1/Akt/mTOR pathway is also involved in miR-646-mediated EMT regulation. Taken together, these findings reveal the novel EMT-suppressive function of miR-646, which might potentially be applied in a novel strategy for targeting of the EMT.

\section{ACKNOWLEDGEMENTS}

This study was supported by grants from the National Natural Science Funds of China (81672875), 'President Foundation of Nanfang Hospital, Southern Medical University' (2012B009, 2013Z007), and high-level topic-matching funds of Nanfang Hospital (201347 and G201227). Guangdong Provincial Key Laboratory of Gastroenterology, Department of Gastroenterology, Nanfang Hospital, Southern Medical University; Guangzhou Pilot Project of Clinical and Translational Research Center (early gastrointestinal cancer,No.7415696196402).

\section{CONFLICT OF INTEREST}

The authors declare no conflict of interest.

\section{AUTHOR CONTRIBUTIONS}

Conceived and designed the experiments: JDW and SDL. Performed the experiments, PZ, WMT, HZ, YQL and YP. Analysed the data: PZ, GNL and XTH. Contributed reagents/ materials/analysis tools: JJZ, GL, AML, YB, YC, YXR, GXL and YDW. Wrote the paper, PZ. All authors were involved in writing the paper and had final approval of the submitted and published versions.

\section{REFERENCES}

Calin GA, Croce CM (2006) MicroRNA-cancer connection: the beginning of a new tale. Cancer Res 66: 7390-7394.

Chang L, Graham PH, Hao J, Ni J, Bucci J, Cozzi PJ, Kearsley JH, Li Y (2013) Acquisition of epithelial-mesenchymal transition and cancer stem cell 
phenotypes is associated with activation of the PI3K/Akt/mTOR pathway in prostate cancer radioresistance. Cell Death Dis 4: e875.

Dong P, Ihira K, Xiong Y, Watari H, Hanley SJ, Yamada T, Hosaka M, Kudo M, Yue J, Sakuragi N (2016) Reactivation of epigenetically silenced miR-124 reverses the epithelial-to-mesenchymal transition and inhibits invasion in endometrial cancer cells via the direct repression of IQGAP1 expression. Oncotarget 7: 20260-20270.

Dwivedi SK, Mustafi SB, Mangala LS, Jiang D, Pradeep S, Rodriguez-Aguayo C, Ling H, Ivan C, Mukherjee P, Calin GA, Lopez-Berestein G, Sood AK, Bhattacharya R (2016) Therapeutic evaluation of microRNA-15a and microRNA-16 in ovarian cancer. Oncotarget 7: 15093-15104.

Fu W, Tao T, Qi M, Wang L, Hu J, Li X, Xing N, Du R, Han B (2016) MicroRNA-132/212 upregulation inhibits TGF- $\beta$-mediated epithelialmesenchymal transition of prostate cancer cells by targeting SOX4. Prostate 76: 1560-1570.

Garry DJ, Meeson A, Elterman J, Zhao Y, Yang P, Bassel Duby R, Williams RS (2000) Myogenic stem cell function is impaired in mice lacking the forkhead/winged helix protein MNF. Proc Natl Acad Sci USA 97: 5416-5421.

Hou LK, Yu Y, Xie YG, Wang J, Mao JF, Zhang B, Wang X, Cao XC (2016) MiR-340 and ZEB1 negative feedback loop regulates TGF- $\beta$ - mediated breast cancer progression. Oncotarget 7: 26016-26026.

Huang JY, Zhang K, Chen DQ, Chen J, Feng B, Song H, Chen Y, Zhu Z, Lu L, De W, Wang R, Chen LB (2015) MicroRNA-451: epithelial-mesenchymal transition inhibitor and prognostic biomarker of hepatocelluar carcinoma. Oncotarget 6: 18613-18630.

Hur K, Toiyama Y, Takahashi M, Balaguer F, Nagasaka T, Koike J, Hemmi H, Koi M, Boland CR, Goel A (2013) MicroRNA-200c modulates epithelialto- mesenchymal transition (EMT) in human colorectal cancer metastasis. Gut 62: 1315-1326.

Ibarrola-Villava M, Llorca-Cardeñosa MJ, Tarazona N, Mongort C, Fleitas T, Perez-Fidalgo JA, Roselló S, Navarro S, Ribas G, Cervantes A (2015) Deregulation of ARID1A, CDH1, cMET and PIK3CA and target-related microRNA expression in gastric cancer. Oncotarget 6: 26935-26945.

Kaneko M, Morimura K, Nishikawa T, Wanibuchi H, Takada N, Osugi H, Kinoshita H, Fukushima S (2002) Different genetic alterations in rat forestomach tumors induced by genotoxic and non-genotoxic carcinogens. Carcinogenesis 23: 1729-1735.

Kim NH, Kim HS, Li XY, Lee I, Choi HS, Kang SE, Cha SY, Ryu JK, Yoon D, Fearon ER, Rowe RG, Lee S, Maher CA, Weiss SJ, Yook JI (2011) A p53/ miRNA-34 axis regulates Snaill-dependent cancer cell epithelialmesenchymal transition. J Cell Biol 195: 417-433.

Li B, Liu L, Li X, Wu L (2015) miR-503 suppresses metastasis of hepatocellular carcinoma cell by targeting PRMT1. Biochem Biophys Res Commun 464: 982-987.

Liu C, Guan H, Wang Y, Chen M, Xu B, Zhang L, Lu K, Tao T, Zhang X, Huang Y (2015) miR-195 inhibits EMT by targeting FGF2 in prostate cancer cells. PLoS One 10: e0144073.

Li W, Liu M, Feng Y, Xu YF, Huang YF, Che JP, Wang GC, Yao XD, Zheng JH (2014) Downregulated miR-646 in clear cell renal carcinoma correlated with tumour metastasis by targeting the nin one binding protein (NOB1). Br J Cancer 111: 1188-1200.

Lu J, Getz G, Miska EA, Alvarez-Saavedra E, Lamb J, Peck D, Sweet-Cordero A, Ebert BL, Mak RH, Ferrando AA, Downing JR, Jacks T, Horvitz HR, Golub TR (2005) MicroRNA expression profiles classify human cancers. Nature 435: 834-838.

Martello G, Rosato A, Ferrari F, Manfrin A, Cordenonsi M, Dupont S, Enzo E, Guzzardo V, Rondina M, Spruce T, Parenti AR, Daidone MG, Bicciato S, Piccolo S (2010) A microRNA targeting dicer for metastasis control. Cell 141: 1195-1207.

Pan Y, Chen Y, Ma D, Ji Z, Cao F, Chen Z, Ning Y, Bai C (2016) miR-646 is a key negative regulator of EGFR pathway in lung cancer. Exp Lung Res 42: 286-295.

Peng Y, Zhang P, Huang X, Yan Q, Wu W, Xie R, Wu Y, Zhang M, Nan Q, Zhao J, Li A, Xiong J, Ren Y, Yang B, Chen Y, Liu S, Wang J (2016) Direct regulation of FOXK1 by C-jun promotes proliferation, invasion and metastasis in gastric cancer cells. Cell Death Dis 7: e2480.

Shah MA, Ajani JA (2010) Gastric cancer-an enigmatic and heterogeneous disease. JAMA 303: 1753-1754.
Stock M, Otto F (2005) Gene deregulation in gastric cancer. Gene 360: $1-19$.

Sun XH, Geng XL, Zhang J, Zhang C (2015) miRNA-646 suppresses osteosarcoma cell metastasis by downregulating fibroblast growth factor 2 (FGF2). Tumour Biol 36: 2127-2134.

Torii A, Kodera Y, Uesaka K, Hirai T, Yasui K, Morimoto T, Yamamura Y, Kato T, Hayakawa T, Fujimoto N, Kito T (1997) Plasma concentration of matrix metalloproteinase 9 in gastric cancer. Br J Surg 84: 133-136.

Wagner AD, Moehler M (2009) Development of targeted therapies in advanced gastric cancer: promising exploratory steps in a new era. Curr Opin Oncol 21: 381-385.

Wang H, Zhang C, Xu L, Zang K, Ning Z, Jiang F, Chi H, Zhu X, Meng Z (2016) Bufalin suppresses hepatocellular carcinoma invasion and metastasis by targeting HIF- $1 \alpha$ via the PI3K/AKT/mTOR pathway. Oncotarget 7: 20193-20208.

Wang J, Yang Y, Xia HH, Gu Q, Lin MC, Jiang B, Peng Y, Li G, An X, Zhang Y, Zhuang Z, Zhang Z, Kung HF, Wong BC (2007) Suppression of FHL2 expression induces cell differentiation and inhibits gastrointestinal carcinogenesis. Gastroenterology 132: 1066-1076.

Wang Q, Li X, Zhu Y, Yang P (2014) MicroRNA-16 suppresses epithelialmesenchymal transition-related gene expression in human glioma. Mol Med Rep 10: 3310-3314.

Wang W, Li X, Lee M, Jun S, Aziz KE, Feng L, Tran MK, Li N, McCrea PD, Park JI, Chen J (2015) FOXKs promote Wnt/ $\beta$ - catenin signaling by translocating DVL into the nucleus. Dev Cell 32: 707-718.

Wu M, Wang J, Tang W, Zhan X, Li Y, Peng Y, Huang X, Bai Y, Zhao J, Li A, Chen C, Chen Y, Peng H, Ren Y, Li G, Liu S, Wang J (2016a) FOXK1 interaction with FHL2 promotes proliferation, invasion and metastasis in colorectal cancer. Oncogenesis 5: e271.

Wu Y, Peng Y, Wu M, Zhang W, Zhang M, Xie R, Zhang P, Bai Y, Zhao J, Li A, Nan Q, Chen Y, Ren Y, Liu S, Wang J (2016b) Oncogene FOXK1 enhances invasion of colorectal carcinoma by inducing epithelialmesenchymal transition. Oncotarget 7: 51150-51162.

Wu Y, Xie R, Liu X, Wang J, Peng Y, Tang W, Wu M, Zhang P, Ba Y, Zhao J, Li A, Nan Q, Chen Y, Liu S, Wang J (2016c) Knockdown of FOXK1 alone or in combination with apoptosis-inducing 5-FU inhibits cell growth in colorectal cancer. Oncol Rep 36: 2151-2159.

Wu Z, Li X, Cai X, Huang C, Zheng M (2016d) miR-497 inhibits epithelial mesenchymal transition in breast carcinoma by targeting Slug. Tumour Biol 37: 7939-7950.

Yang MH, Wu MZ, Chiou SH, Chen PM, Chang SY, Liu CJ, Teng SC, Wu KJ (2008) Direct regulation of TWIST by HIF-1alpha promotes metastasis. Nat Cell Biol 10: 295-305.

Yang Q, Kong Y, Rothermel B, Garry DJ, Bassel-Duby R, Williams RS (2000) The winged-helix/forkhead protein myocyte nuclear factor beta (MNFbeta) forms a co-repressor complex with mammalian $\sin 3$ B. Biochem J 345: 335-343.

Ying H, Lyu J, Ying T, Li J, Jin S, Shao J, Wang L, Xu H (2014) Risk miRNA screening of ovarian cancer based on miRNA functional synergistic network. J Ovarian Res 7: 9

Yolanda LV, Sergio PD, Hugo ES, Isabel AF, Rafael BZ, Aldo TD, Gonzalo CR (2015) Gastric cancer progression associated with local humoral immune responses. BMC Cancer 15: 924.

Yu T, Li J, Yan M, Liu L, Lin H, Zhao F, Sun L, Zhang Y, Cui Y, Zhang F, Li J, He X, Yao M (2015) MicroRNA-193a-3p and -5p suppress the metastasis of human non-small-cell lung cancer by downregulating the ERBB4/ PIK3R3/mTOR/S6K2 signaling pathway. Oncogene 34: 413-423.

Zhou JN, Zeng Q, Wang HY, Zhang B, Li ST, Nan X, Cao N, Fu CJ, Yan XL, Jia YL, Wang JX, Zhao AH, Li ZW, Li YH, Xie XY, Zhang XM, Dong Y, Xu YC, He LJ, Yue W, Pei XT (2015) MicroRNA-125b attenuates epithelial-mesenchymal transitions and targets stem-like liver cancer cells through small mothers against decapentaplegic 2 and 4. Hepatology 62: 801-815.

This work is published under the standard license to publish agreement. After 12 months the work will become freely available and the license terms will switch to a Creative Commons AttributionNonCommercial-Share Alike 4.0 Unported License. 